\title{
Influence of an external electric field on the potential-energy surface of alkali-metal-decorated $\mathbf{C}_{60}$
}

\author{
Deb Sankar De, ${ }^{1}$ Santanu Saha, ${ }^{1}$ Luigi Genovese, ${ }^{2}$ and Stefan Goedecker ${ }^{1}$ \\ ${ }^{1}$ Department of Physics, Universität Basel, Klingelbergstr. 82, 4056 Basel, Switzerland \\ ${ }^{2}$ Univ. Grenoble Alpes, CEA, INAC-SP2M, L_Sim, F-38000, Grenoble, France
}

(Received 19 March 2018; published 4 June 2018)

\begin{abstract}
We present a fully $a b$ initio, unbiased structure search of the configurational space of decorated $\mathrm{C}_{60}$ fullerenes in the presence of an electric field. We observed that the potential-energy surface is significantly perturbed by an external electric field and that the energetic ordering of low-energy isomers differs with and without electric field. We identify the energetically lowest configuration for a varying number of decorating atoms $(1 \leqslant n \leqslant 12)$ for $\mathrm{Li}$ and $(1 \leqslant n \leqslant 6)$ for $\mathrm{K}$ on the $\mathrm{C}_{60}$ surface at different electric-field strengths. Using the correct geometric ground state in the electric field for the calculation of the dipole we obtain better agreement with the experimentally measured values than previous calculations based on the ground state in absence of an electric field. Since the lowest-energy structures are typically nearly degenerate in energy, a combination of different structures is expected to be found at room temperature. The experimentally measured dipole is therefore also expected to contain significant contributions from several low-energy structures.
\end{abstract}

DOI: 10.1103/PhysRevA.97.063401

\section{INTRODUCTION}

For the past three decades, fullerenes played a prominent role in the field of nanoscience and nanotechnology because of their unusual chemical and physical properties. By doping bulk fullerenes with alkali metals, one can obtain intercalation compounds with interesting physical properties such as superconductivity [1-4]. Alkaline and alkaline-earth-metals coated $\mathrm{C}_{60}$ and carbon nanotubes has been suggested as a potential hydrogen storage material [5-10]. Recently, it has been suggested that applying an electric field vertical to the surface of the metal decorated graphene (or other systems) enhances the hydrogen adsorption [11-14]. Moreover, this process is a reversible process and hydrogen can be easily desorbed by applying an opposite electric field.

Electric dipole measurements provide a convenient way to determine the electronic and geometrical structures of metal decorated $\mathrm{C}_{60}[15,16]$. Structural changes induced by an electric field have been observed for various systems such as polycrystalline pentacene-based organic transistors [17], pentacene single crystals [18], and polar or nonpolar molecules in a dodecahedral water cage [19]. Uncoated fullerenes are prone to structural changes in the gas phase in the presence of an electric field [20,21].

Rayane et al. measured the polarizibility and the permanent electric dipole moment of isolated $\mathrm{KC}_{60}$ molecules using molecular-beam deflection [22]. Their results suggested that the polarizibility of $\mathrm{KC}_{60}$ is induced by the free skating of the potassium atom on the $\mathrm{C}_{60}$ surface. Antoine et al. extended the work to different alkali-metal decorated $\mathrm{C}_{60}$ 's [23]. They observed that the dipole moment increases steadily for the elements from top to bottom of the first column of the Periodic Table. A strong charge transfer between a single alkali-metal atom and the $\mathrm{C}_{60}$ cage has been reported resulting in a large electric dipole moment $[23,24]$. Dugourd et al. observed a high electric susceptibility of $\mathrm{Na}_{n} \mathrm{C}_{60}(n$ is the number of $\mathrm{Na}$ atoms) [25]. The results were attributed to a high electric dipole arising from the aggregation of $\mathrm{Na}_{n}$ on the $\mathrm{C}_{60}$. Antoine et al. also measured electric susceptibilities for $\mathrm{Li}_{n} \mathrm{C}_{60}$ and $\mathrm{Na}_{n} \mathrm{C}_{60}$ clusters containing up to $n=20$ alkali-metal atoms [26]. From the experimental data, they concluded that for more than seven $\mathrm{Na}$ atoms all atoms aggregate into a single cluster, whereas for $\mathrm{Li}$ not all atoms on $\mathrm{C}_{60}$ participate in such a clustering if more than 12 atoms are present.

Antonio et al. also explained their experimental results by using a parametrized many-body force field in combination with basin hopping [27] to find the most stable structure for $\mathrm{Li}$ and $\mathrm{Na}$ decorated $\mathrm{C}_{60}$ [26]. However, the calculated electrostatic dipole moments for the previously known ground states for different $n$ did not match with the experimental results. Rabilloud et al. computed the dipole moments of $\mathrm{C}_{60}$ structures decorated with a few $\mathrm{Li}$ and $\mathrm{Na}$ atoms at the DFT level [28]. By comparing the dipole moments with the experimental value, it was suggested that the configurations whose dipole moments are closer to the experimental values might be present in the experiment, even though they are energetically higher than other structures.

In this work we present an unbiased PES scan for Li and $\mathrm{K}$ decorated $\mathrm{C}_{60}$ at the DFT level in the presence of an electric field. The wavelet basis set of the BIGDFT code is highly suitable for such a calculation. One can use free boundary conditions which allow for a constant electric field throughout the simulation box. In addition the basis set is systematic and adapts itself fully to the distortion of the wave function induced by the electric field. In this way results of identical high quality can be obtained with and without electric field.

In previous work, we have observed that $\mathrm{Li}$ and $\mathrm{K}$ atoms prefer to homogeneously distribute over $\mathrm{C}_{60}$ up to 12 and 6 atoms, respectively, in the absence of an external electric field [29]. For this reason we have limited the present PES scan to this maximum number of atoms. 


\section{METHODS}

The unbiased structural prediction of decorated fullerenes in an external electric field was conducted using the minima hopping method [30-32], as implemented in the BIGDFT package [33]. MHM aims to search the global minimum on the potential-energy surface while gradually exploring the other low-energy structures. The efficiency of the MHM method is due to the exploitation of the Bell-Evans-Polanyi principle for molecular dynamics [34]. This method has been successfully applied to many different systems for global geometry optimization [35-43].

The BIGDFT code is a density-functional code using a systematic Daubechies wavelet basis set [44]. The PerdewBurke-Ernzerhof exchange-correlation functional [45] as implemented in the LibXC library [46] was used together with PBE dual-space Gaussian pseudopotentials with a nonlinear core correction [47-49]. Tight electronic parameters were chosen such that total energy differences were converged to at least $10^{-4} \mathrm{eV}$ for all stable configurations. The forces in geometry relaxations were converged to below $1 \mathrm{meV} / \AA$.

\section{RESULTS AND DISCUSSION}

\section{A. Single $\mathrm{Li}$ and $\mathrm{K}$ atom on $\mathrm{C}_{60}$}

From our previous calculations, we know that alkali-metal atoms, except for Li, prefer to adsorb on the hexagonal site [29]. The potential-energy surface can be altered by applying an external electric field and desired configurations can be stabilized. In the presence of a sufficiently strong electric field, the pentagonal site can however become energetically preferable, as shown in Fig. 1.

Alkali-metal atoms make strong ionic bonds with $\mathrm{C}_{60}$ acting as an electron donor for the $\mathrm{C}_{60}[23,24]$. The donated charge is strongly localized on the carbon that is closest to the metal atom. As the strength of the electric field increases, the positively charged alkali-metal atom moves in the direction of the electric field, while the negatively charged $\mathrm{C}_{60}$ moves in the

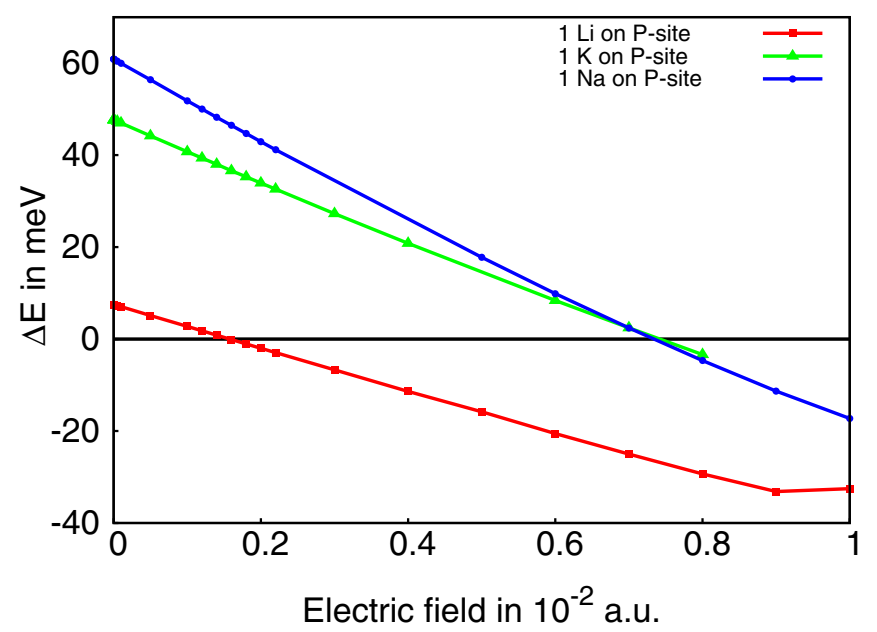

FIG. 1. Energy of the pentagonal site with respect to the hexagonal site for one $\mathrm{Li}, \mathrm{K}$, or $\mathrm{Na}$ atom on $\mathrm{C}_{60}$ as a function of the electric-field strength.

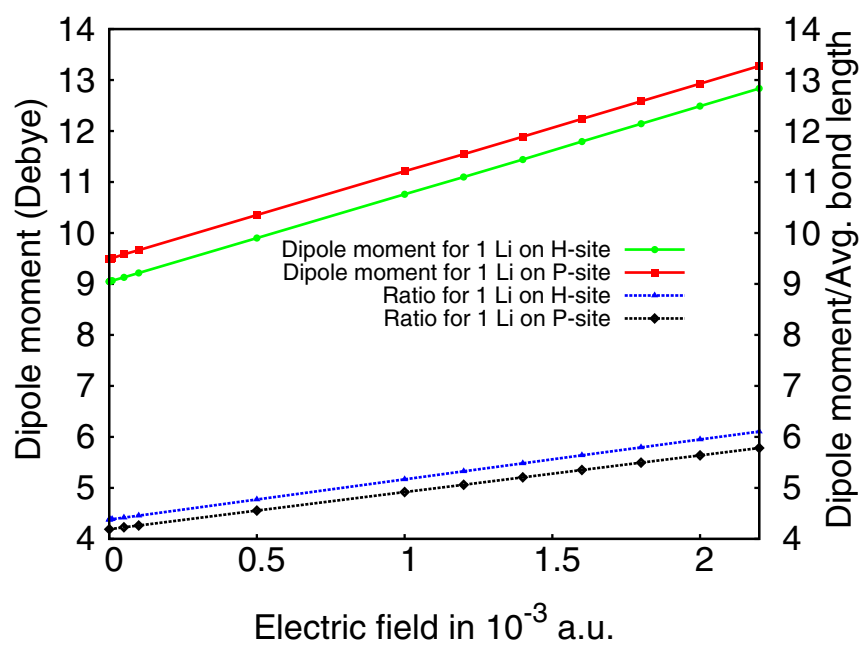

FIG. 2. Dipole moment and the ratio of dipole moment and average bond length between the alkali-metal atom and the carbon atom from the $\mathrm{C}_{60}$ vs electric field for one $\mathrm{Li}$ atom on the surface of $\mathrm{a} \mathrm{C}_{60}$.

opposite direction. This increase in the distance between the positive and negative charge leads to a mainly linear increase of the dipole moment with respect to the field strength since the charges remain more or less constant as shown in Fig. 2.

\section{B. $\mathrm{Li}$ and $\mathrm{K}$ on the fullerene surface}

In this section, we provide further insight into the behavior of $\mathrm{C}_{60}$ 's decorated with more than one $\mathrm{Li}$ in the presence of an electric field. The number of atoms on the $\mathrm{C}_{60}$ surface is stepwise increased from 2 to 12 for $\mathrm{Li}$ and from 2 to 6 for $\mathrm{K}$. The electric field used for these calculations was $5 \times 10^{-5}$ atomic unit (a.u.), which is comparable to the strength of the experimentally applied electric field $\left(7 \times 10^{2} \mathrm{~V} / \mathrm{m}\right)$ [26]. We also performed another MH run with an electric field of $1 \times 10^{-3}$ a.u., which corresponds to a strong experimental field reached for instance in the tip of a scanning microscope [50]. We started our calculations with the lowest-energy structure at zero electric field. Figure 3 presents two energetically quasidegenerate metastable structures in the absence of an electric field. In one structure, one of the two Li atoms is on $\mathrm{P}$ site [Fig. 3(a)], whereas both Li atoms are on $\mathrm{H}$ site in the other structure [Fig. 3(b)]. At low electric field $\left(5 \times 10^{-5}\right.$ a.u. $)$ they are still energetically degenerate. As we increase the electric field, the energy difference between these structures increases (a)

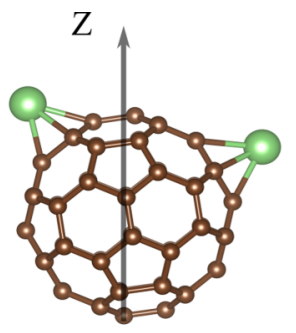

(b)

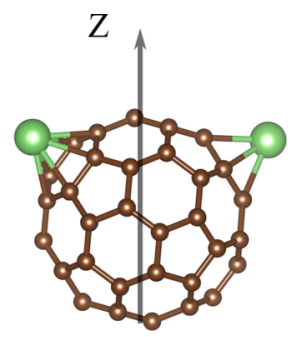

FIG. 3. Two isomers [(a) and (b)] found for an electric field of $5 \times 10^{-5}$ a.u. The electric field is along the $Z$ direction. 


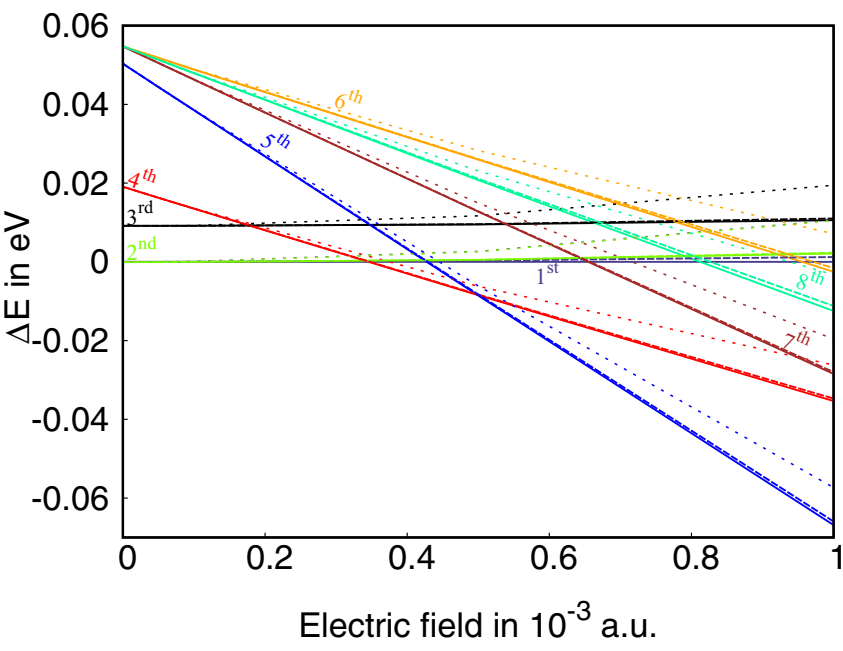

FIG. 4. Solid lines show the energy of eight isomers of $\mathrm{Li}_{2} \mathrm{C}_{60}$ as a function of the applied electric field, whereas the dotted lines show their energies obtained from the first-order perturbation theory and the dashed lines give their energies obtained from the secondorder perturbation theory. All energies are calculated with respect to the lowest-energy structure at zero electric field. The dashed lines overlap with the solid lines. The numbering of the structures in the plot corresponds to the energy ordering of the structures at zero electric field. First and second structure is energetically the same at the low electric fields.

strongly(fourth and fifth lowest-energy structure of Fig. 4). A similar situation arises for more than two Li atoms.

To establish the energetic ordering of the structures as a function of the field strength, we relaxed the 20 lowest-energy structures from minima hopping runs at different electric-field strengths and the first eight of them are plotted in Fig. 4. The two structures that were initially the lowest-energy structures are not any more energetically favorable at a strength of $4 \times 10^{-4}$ a.u., while other structures are lowered in energy. Different energetic orderings at different electric fields are also observed for more than $2 \mathrm{Li}$ atoms on the $\mathrm{C}_{60}$ surface. In addition to the total energies $E_{\text {tot }}$ calculated consistently in the electric field, we have plotted in Fig. 4 also the energies from first- and second-order perturbation theory, which are given by

$$
E=E(\mathcal{E}=0)-\sum_{i} P_{i} \mathcal{E}_{i}-\frac{1}{2} \sum_{i} \sum_{j} \alpha_{i j} \mathcal{E}_{i} \mathcal{E}_{j}
$$

where $\mathcal{E}$ is the applied electric field, $P_{i}$ and $\alpha_{i j}$ are the static dipole moment and polarizibility, respectively, and $E(\mathcal{E}=0)$ is the energy of the system without electric field. At low electric field the dotted and solid lines overlap, but at higher electric-field strengths they deviate, showing that first-order perturbation theory cannot predict the correct energetic ordering for strong experimental fields. This is because the change in dipole moment is not considered. As we add the second-order perturbation term to the energy, the energies almost overlap with the actual energy. This shows that the second-order variation in energy due to the first-order variation of the dipole moment (i.e., linear polarizibility term) is necessary for an accurate description of this system in the strong electric fields.

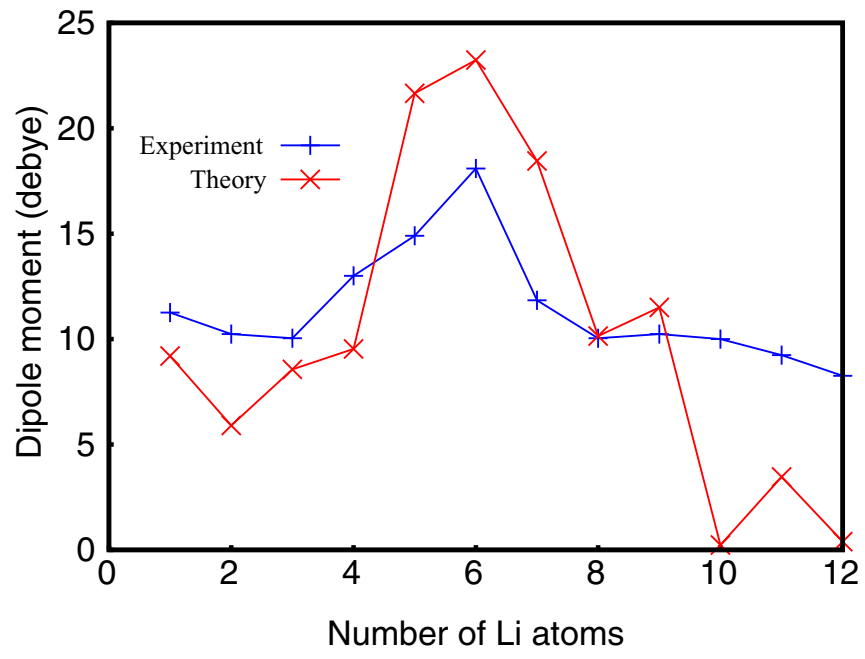

FIG. 5. Theoretical and experimental [26] dipole moments for the lowest-energy structure for different number of $\mathrm{Li}$ atoms decorated $\mathrm{C}_{60}$. The experimental data is obtained from the article by Antonio et al. [26]. For theoretical calculation, the applied electric field is $5 \times 10^{-5}$ a.u. and, for experiment, $3 \times 10^{-5}$ a.u.

The dipole moments for different numbers of Li atoms are plotted in Fig. 5 for a field strength of $5 \times 10^{-5}$ a.u. The experimental values [26] are intrinsically Boltzmann averages over low-energy configurations, whereas the theoretical value is only obtained from the lowest-energy configuration, because it would be computationally too expensive to calculate the energies of a large number of structures for many different field strengths. If there is an energetically degenerate state for a particular number of $\mathrm{Li}$ atoms, we have taken the one with the highest dipole moment. In contrast to the experimental results which were obtained at room temperature, our results are at zero temperature, since it would also be computationally too expensive to calculate the required large number of free energies. In spite of these differences between the experimental and theoretical dipole moments, Fig. 5 shows similar trends such as a peak of the dipole moment for six Li atoms.

In order to study the stability of some selected configurations at finite temperature, we calculated free energies. We calculated in the standard way [51] the vibrational frequencies $\omega_{i}$ and the zero point energy $E_{Z P}$ to obtain the free energy $F$,

$$
F=E_{0}-E_{\mathrm{ZP}}+k_{\mathrm{B}} T \sum_{i} \ln \left[\exp \left(\frac{\hbar \omega_{\mathrm{i}}}{k_{\mathrm{B}} T}\right)-1\right] \text {. }
$$

Figure 6 shows that the lowest-energy structure at zero temperature is still the lowest in energy at room temperature and remains highly populated. There is, however, a high probability of populating the higher-energy structures (Fig. 7). The dipole moment for fifth and sixth configuration is quite high $(\sim 10$ Debye). These structures will be present during the measurement of dipole moment at room temperature and contribute to the total dipole moment. In Fig. 8, we have calculated the total dipole moment as a sum of the dipole moments of different structures weighted by their Boltzmann probability factors. As we include more isomers the total dipole moment tends towards the experimental value (10.2 Debye) at room temperature. Reproducing exactly the experimental value is, 


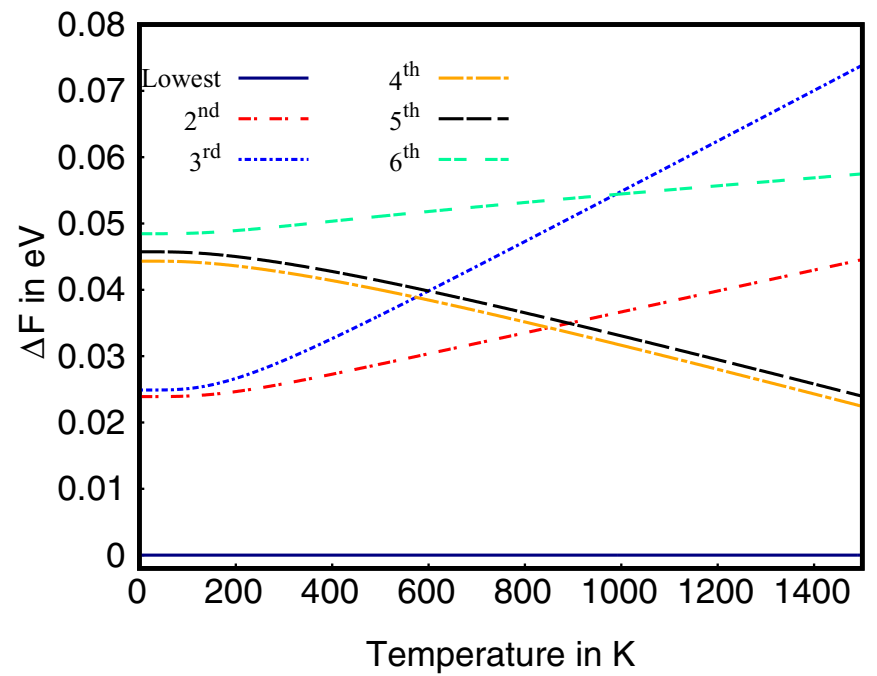

FIG. 6. Free energy for the six lowest-energy configurations. The free energies are plotted with respect to the energy of the lowestenergy configuration at $0 \mathrm{~K}$. The applied electric field is $5 \times 10^{-5}$ a.u.

however, elusive since the intrinsic errors of density-functional theory are larger than $k_{B} T$ at room temperature and hence it is not possible to obtain reliable Boltzmann probabilities.

$\mathrm{C}_{60}$ does not have double bonds within the pentagonal rings. Hence one electron is missing to obtain an additional stabilization by aromaticity. As a result, $\mathrm{C}_{60}$ behaves like an electron deficient alkene and readily reacts with electron rich species. The estimated electron affinity and ionization potential values for $\mathrm{C}_{60}$ are $2.7 \mathrm{eV}$ and $7.8 \mathrm{eV}$, respectively [52]. The neutral $\mathrm{C}_{60}$ can take six extra electron to achieve higher aromaticity. Even an additional six electrons can be accommodated [53]. Such a $\mathrm{C}_{60}^{n-}$ (where $\left.n=2,3,4\right)$ structure is short lived [54,55], but can be stabilized by an $\mathrm{Li}^{+}[28,56]$. This explains the experimentally observed [57] and theoretically confirmed [29] homogeneous absorption of up to $12 \mathrm{Li}$ atoms on $\mathrm{C}_{60}$. Hence,

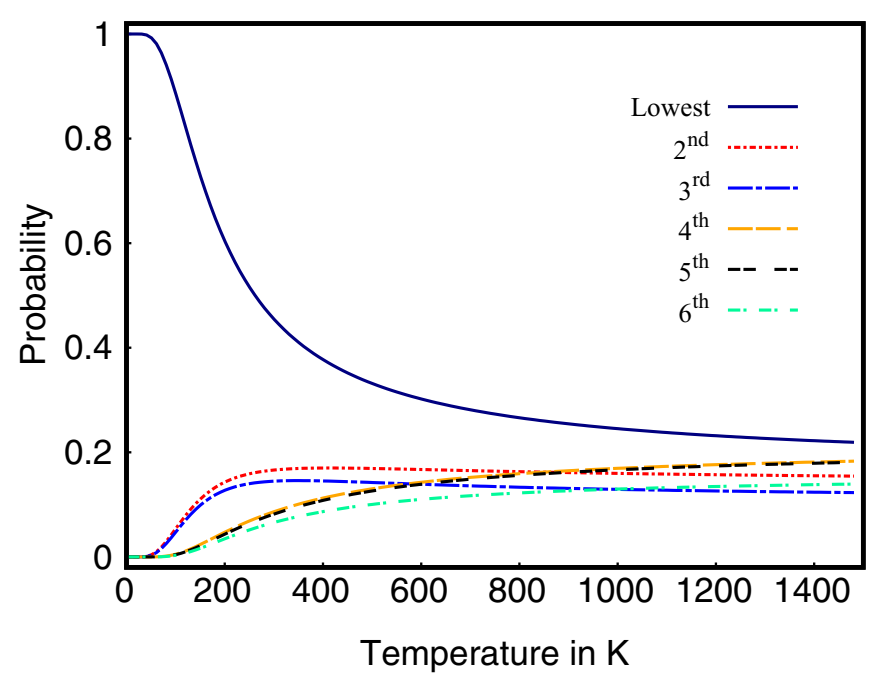

FIG. 7. Normalized Boltzmann factors as a function of temperature for the six low-energy configurations. The applied electric field is $5 \times 10^{-5}$ a.u.

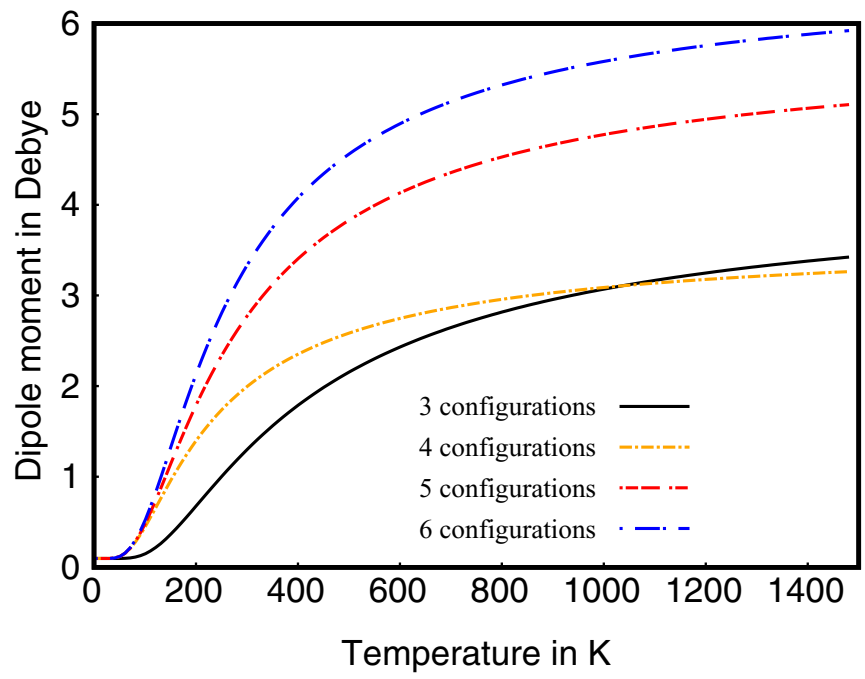

FIG. 8. Dipole moment of the system at different temperatures obtained by weighting the dipole of the individual structures by their Boltzmann factor. The applied electric field is $5 \times 10^{-5}$ a.u.

to alter this distribution pattern, a high electric field is required. The configurations that are provided in our previous work [29] are the most stable configurations up to a field strength of $1 \times 10^{-3}$ a.u. for $\mathrm{Li}_{4}$ and $3 \times 10^{-3}$ a.u. for $\mathrm{Li}_{6}$ and $\mathrm{Li}_{11}$. For even stronger fields the $\mathrm{Li}$ atoms are detached from the $\mathrm{C}_{60}$, but clustering is never observed.

The PES of the $\mathrm{K}$ atom decorated $\mathrm{C}_{60}$ slowly changes with an increasing electric field. Table II contains the energy ordering for the first 10 lowest-energy structures of $\mathrm{K}_{2} \mathrm{C}_{60}$ at different electric fields. The lowest-energy structure which is observed at very low electric field is different from the lowest-energy structure in the absence of an electric field. As the number of $\mathrm{K}$ atom is increased, the structures are even more stable and require high electric field to alter the energy ordering, for example, for $\mathrm{K}_{6} \mathrm{C}_{60}$ and the PES alters at the electric field of $1 \times 10^{-3}$ a.u.

In Table I, we have shown the dipole moment of the lowest-energy structures of $\mathrm{K}$ decorated $\mathrm{C}_{60}$ at the electric field of $5 \times 10^{-5}$ a.u. Our calculated dipole moment for one $\mathrm{K}$ atom on $\mathrm{C}_{60}$ is 15 Debye in an electric field of $5 \times 10^{-5}$ a.u. The ATD profile with an electric field of $1.5 \times 10^{7} \mathrm{~V} / \mathrm{m}$ is in good agreement with the profile simulated for a permanent (and rigid) dipole moment of 17.7 Debye, which is very close to the theoretical value $[22,24]$. If we increase the number of $\mathrm{K}$ atoms, many energetically degenerate structures appear as we increase the applied electric field (Table II). We have not

TABLE I. Dipole moment of the lowest-energy structure for different number of $\mathrm{K}$ atoms decorated $\mathrm{C}_{60}$. The applied electric field is $5 \times 10^{-5}$ a.u.

\begin{tabular}{lcccccc}
\hline \hline $\begin{array}{l}\text { Number of } \\
\text { K atoms }\end{array}$ & 1 & 2 & 3 & 4 & 5 & 6 \\
\hline $\begin{array}{l}\text { Dipole } \\
\text { moment } \\
\text { (Debye) }\end{array}$ & 15.38 & 10.19 & 5.73 & 0.35 & 11.09 & 0.21 \\
\hline \hline
\end{tabular}


TABLE II. Energy of the first $10 \mathrm{~K}_{2} \mathrm{C}_{60}$ structures at different electric field. The energies are given with respect to the lowest-energy structure at that particular electric field in $\mathrm{eV}$.

\begin{tabular}{lcccc}
\hline \hline $\begin{array}{l}\text { Structure } \\
\text { ordering }\end{array}$ & $\begin{array}{c}5 \times 10^{-5} \\
\text { a.u. }\end{array}$ & $\begin{array}{c}1 \times 10^{-4} \\
\text { a.u. }\end{array}$ & $\begin{array}{c}5 \times 10^{-4} \\
\text { a.u. }\end{array}$ & $\begin{array}{c}1 \times 10^{-3} \\
\text { a.u. }\end{array}$ \\
\hline 1 & 0.000 & 0.037 & 0.055 & 0.096 \\
2 & 0.009 & 0.074 & 0.000 & 0.000 \\
3 & 0.019 & 0.097 & 0.107 & 0.221 \\
4 & 0.021 & 0.104 & 0.139 & 0.289 \\
5 & 0.068 & 0.000 & 0.106 & 0.028 \\
6 & 0.068 & 0.000 & 0.107 & 0.026 \\
7 & 0.072 & 0.095 & 0.113 & 0.127 \\
8 & 0.088 & 0.164 & 0.158 & 0.252 \\
9 & 0.119 & 0.201 & 0.263 & 0.438 \\
10 & 0.138 & 0.201 & 0.179 & 0.208 \\
\hline \hline
\end{tabular}

found the experimental dipole moment for $\mathrm{C}_{60}$ with more than one $\mathrm{K}$ atom in the literature.

We also investigated if all $\mathrm{C}_{60}$ molecules in a group will have exactly the same number of $\mathrm{K}$ atoms on the surface or whether there may be a redistribution of decorating $\mathrm{K}$ atoms on $\mathrm{C}_{60}$ molecules. We checked, for example, whether two $\mathrm{K}_{4} \mathrm{C}_{60}$ are energetically more favorable than one $\mathrm{K}_{3} \mathrm{C}_{60}$ and one $\mathrm{K}_{5} \mathrm{C}_{60}$. We calculated the energy difference for those two cases using the following equation:

$$
E_{\text {diff }}=2 \times E_{K_{4} C_{60}}-E_{K_{3} C_{60}}-E_{K_{5} C_{60}} .
$$

We found a tiny energy difference $\left(E_{\text {diff }}\right)$ of $0.14 \mathrm{eV}$, which indicates that they are energetically degenerate. Hence two $\mathrm{K}_{4} \mathrm{C}_{60}$ structures are equally likely to occur than one $\mathrm{K}_{3} \mathrm{C}_{60}$ and one $\mathrm{K}_{5} \mathrm{C}_{60}$. Experimentally, however, it is possible to obtain a group of $\mathrm{C}_{60}$ atoms with an identical number of $\mathrm{K}$ atoms $[22,23,26]$.

\section{CONCLUSIONS}

We have explored the PES of $\mathrm{Li}$ and $\mathrm{K}$ decorated $\mathrm{C}_{60}$ in the presence of electric fields of varying strength by an unbiased search method at the DFT level. The experimental field strengths, that can for instance be obtained near the tip in a scanning microscope, induce considerable changes of the PES and alter the energetic ordering of $\mathrm{C}_{60}$ isomers decorated with a small number of metal atoms. Some structures which are metastable in the absence of an electric field can become ground states by varying the strength of the electric field. In this way one can switch between two structures by increasing or weakening the electric field. A correct calculation of measured dipoles requires one to use all those configurations which are the lowest in energy at the given electric field. Since these configurations are frequently virtually degenerate in energy, several configurations can make significant contributions to the dipole moment. Accurate results in strong experimental fields cannot be obtained by perturbation theory but require a fully self-consistent electronic structure calculation for the given field strength.

\section{ACKNOWLEDGMENTS}

D.S.D. acknowledges support from the Swiss National Science Foundation-SNF. Computing resources were provided by the CSCS in Lugano under Project No. s707. Calculations were also performed at the sciCORE [58] scientific computing core facility at the University of Basel. This work was done partially within the NCCR MARVEL project.
[1] O. Gunnarsson, Rev. Mod. Phys. 69, 575 (1997).

[2] M. Mitrano, A. Cantaluppi, D. Nicoletti, S. Kaiser, A. Perucchi, S. Lupi, P. Di Pietro, D. Pontiroli, M. Riccò, S. R. Clark et al., Nature (London) 530, 461 (2016).

[3] H. Takeya, T. Konno, C. Hirata, T. Wakahara, K. Miyazawa, T. Yamaguchi, M. Tanaka, and Y. Takano, J. Phys.: Condens. Matter 28, 354003 (2016).

[4] M. Kim, Y. Nomura, M. Ferrero, P. Seth, O. Parcollet, and A. Georges, Phys. Rev. B 94, 155152 (2016).

[5] T. Yildirim, J. Íñiguez, and S. Ciraci, Phys. Rev. B 72, 153403 (2005).

[6] K. Chandrakumar and S. K. Ghosh, Nano Lett. 8, 13 (2008).

[7] M. Yoon, S. Yang, C. Hicke, E. Wang, D. Geohegan, and Z. Zhang, Phys. Rev. Lett. 100, 206806 (2008).

[8] Q. Wang, Q. Sun, P. Jena, and Y. Kawazoe, J. Chem. Theory Comput. 5, 374 (2009).

[9] H. Lee, J. Ihm, M. L. Cohen, and S. G. Louie, Phys. Rev. B 80, 115412 (2009).

[10] A. Kaiser, M. Renzler, L. Kranabetter, M. Schwärzler, R. Parajuli, O. Echt, and P. Scheier, Int. J. Hydrogen Energy 42, 3078 (2017).
[11] X. Zhang, C. Tang, and Q. Jiang, Int. J. Hydrogen Energy 41, 10776 (2016).

[12] W. Liu, Y. Zhao, J. Nguyen, Y. Li, Q. Jiang, and E. Lavernia, Carbon 47, 3452 (2009).

[13] S. Shi, J.-Y. Hwang, X. Li, X. Sun, and B. I. Lee, Int. J. Hydrogen Energy 35, 629 (2010).

[14] E. H. Song, S. H. Yoo, J. J. Kim, S. W. Lai, Q. Jiang, and S. O. Cho, Phys. Chem. Chem. Phys. 16, 23985 (2014).

[15] M. Broyer, R. Antoine, E. Benichou, I. Compagnon, P. Dugourd, and D. Rayane, C. R. Phys. 3, 301 (2002).

[16] P. Dugourd, R. Antoine, G. Breaux, M. Broyer, and M. F. Jarrold, J. Am. Chem. Soc. 127, 4675 (2005).

[17] H.-L. Cheng, W.-Y. Chou, C. Kuo, F.-C. Tang, and Y.-W. Wang, Appl. Phys. Lett. 88, 161918 (2006).

[18] K. Kotsuki, S. Obata, and K. Saiki, Langmuir 30, 14286 (2014).

[19] N. D. Gurav, S. P. Gejji, L. J. Bartolotti, and R. K. Pathak, J. Chem. Phys. 145, 074302 (2016).

[20] H. Shen, Int. J. Nanosci. 4, 389 (2005).

[21] J.-y. Sorimachi and S. Okada, Chem. Phys. Lett. 659, 1 (2016).

[22] D. Rayane, R. Antoine, Ph. Dugourd, E. Benichou, A. R. Allouche, M. Aubert-Frécon, and M. Broyer, Phys. Rev. Lett. 84, 1962 (2000). 
[23] R. Antoine, D. Rayane, E. Benichou, P. Dugourd, and M. Broyer, Eur. Phys. J. D 12, 147 (2000).

[24] F. Rabilloud, Comput. Theor. Chem. 964, 213 (2011).

[25] P. Dugourd, R. Antoine, D. Rayane, I. Compagnon, and M. Broyer, J. Chem. Phys. 114, 1970 (2001).

[26] F. Rabilloud, R. Antoine, M. Broyer, I. Compagnon, P. Dugourd, D. Rayane, F. Calvo, and F. Spiegelman, J. Phys. Chem. C 111, 17795 (2007).

[27] J. P. K. Doye and D. J. Wales, Phys. Rev. Lett. 80, 1357 (1998).

[28] F. Rabilloud, J. Phys. Chem. A 114, 7241 (2010).

[29] D. S. De, J. A. Flores-Livas, S. Saha, L. Genovese, and S. Goedecker, Carbon 129, 847 (2018).

[30] S. Goedecker, J. Chem. Phys. 120, 9911 (2004).

[31] B. Schaefer, S. Alireza Ghasemi, S. Roy, and S. Goedecker, J. Chem. Phys. 142, 034112 (2015).

[32] S. Goedecker, W. Hellmann, and T. Lenosky, Phys. Rev. Lett. 95, 055501 (2005).

[33] L. Genovese, A. Neelov, S. Goedecker, T. Deutsch, S. A. Ghasemi, A. Willand, D. Caliste, O. Zilberberg, M. Rayson, A. Bergman, and R. Schneider, J. Chem. Phys. 129, 014109 (2008).

[34] S. Roy, S. Goedecker, and V. Hellmann, Phys. Rev. E 77, 056707 (2008).

[35] M. Amsler, J. A. Flores-Livas, L. Lehtovaara, F. Balima, S. A. Ghasemi, D. Machon, S. Pailhes, A. Willand, D. Caliste, S. Botti et al., Phys. Rev. Lett. 108, 065501 (2012).

[36] M. Amsler, J. A. Flores-Livas, T. D. Huan, S. Botti, M. A. L. Marques, and S. Goedecker, Phys. Rev. Lett. 108, 205505 (2012).

[37] S. Botti, J. A. Flores-Livas, M. Amsler, S. Goedecker, and M. A. L. Marques, Phys. Rev. B 86, 121204(R) (2012).

[38] S. Botti, M. Amsler, J. A. Flores-Livas, P. Ceria, S. Goedecker, and M. A. L. Marques, Phys. Rev. B 88, 014102 (2013).

[39] J. A. Flores-Livas, A. Sanna, and E. Gross, Eur. Phys. J. B 89, 63 (2016).

[40] M. Amsler, J. A. Flores-Livas, M. A. Marques, S. Botti, and S. Goedecker, Eur. Phys. J. B 86, 383 (2013).
[41] B. Schaefer, R. Pal, N. S. Khetrapal, M. Amsler, A. Sadeghi, V. Blum, X. C. Zeng, S. Goedecker, and L.-S. Wang, ACS Nano 8, 7413 (2014).

[42] J. A. Flores-Livas, M. Amsler, T. J. Lenosky, L. Lehtovaara, S. Botti, M. A. L. Marques, and S. Goedecker, Phys. Rev. Lett. 108, 117004 (2012).

[43] J. A. Flores-Livas, M. Amsler, C. Heil, A. Sanna, L. Boeri, G. Profeta, C. Wolverton, S. Goedecker, and E. K. U. Gross, Phys. Rev. B 93, 020508(R) (2016).

[44] I. Daubechies, Ten Lectures on Wavelets (SIAM, Philadelphia, 1992), Vol. 61.

[45] J. P. Perdew, K. Burke, and M. Ernzerhof, Phys. Rev. Lett. 77, 3865 (1996).

[46] M. A. Marques, M. J. Oliveira, and T. Burnus, Comput. Phys. Commun. 183, 2272 (2012).

[47] S. Goedecker, M. Teter, and J. Hutter, Phys. Rev. B 54, 1703 (1996).

[48] A. Willand, Y. O. Kvashnin, L. Genovese, A. Vazquez Mayagoitia, A. K. Deb, A. Sadeghi, T. Deutsch, and S. Goedecker, J. Chem. Phys. 138, 104109 (2013).

[49] C. Hartwigsen, S. Goedecker, and J. Hutter, Phys. Rev. B 58, 3641 (1998).

[50] J. A. Stroscio and D. Eigler, Science 254, 1319 (1991).

[51] J. Ochterski, Thermochemistry in Gaussian (Gaussian, Wallingford, CT, 2000).

[52] A. Rosén and B. Wästberg, J. Chem. Phys. 90, 2525 (1989).

[53] K. E. Geckeler, Advanced Macromolecular and Supramolecular Materials and Processes (Springer, Berlin, 2003).

[54] V. Cammarata, T. Guo, A. Illies, L. Li, and P. Shevlin, J. Phys. Chem. A 109, 2765 (2005).

[55] S. Tomita, J. U. Andersen, H. Cederquist, B. Concina, O. Echt, J. Forster, K. Hansen, B. Huber, P. Hvelplund, J. Jensen et al., J. Chem. Phys. 124, 024310 (2006).

[56] C. Yannouleas and U. Landman, Chem. Phys. Lett. 217, 175 (1994).

[57] U. Zimmermann, N. Malinowski, A. Burkhardt, and T. Martin, Carbon 33, 995 (1995).

[58] http://scicore.unibas.ch/. 